\title{
Using the Two-Burn Escape Maneuver for Fast Transfers in the Solar System and Beyond
}

\author{
Robert B. Adams ${ }^{1}$ \\ National Aeronautics and Space Administration, George C. Marshall Space Flight Center, MSFC, AL \\ 35812 \\ Georgia A. Richardson ${ }^{2}$ \\ University of Alabama in Huntsville, Mechanical and Aerospace Engineering Department, Huntsville, AL \\ 35899
}

The two-burn maneuver to escape the gravitational pull of a central body is described. The maneuver, originally suggested by Hermann Oberth, improves efficiency considerably for a wide range of missions of interest in space exploration and scientific investigation. A clear delineation of when the maneuver is more effective is given, as are methods to extract the most advantage when using the maneuver. Some examples are given of how this maneuver can enable exploration of the outer solar system, near interstellar space, and crewed missions to Mars and beyond. The maneuver has the potential to halve the required infrastructure associated with a crewed mission to Mars and achieve increased solar escape velocities with existing spacecraft technologies.

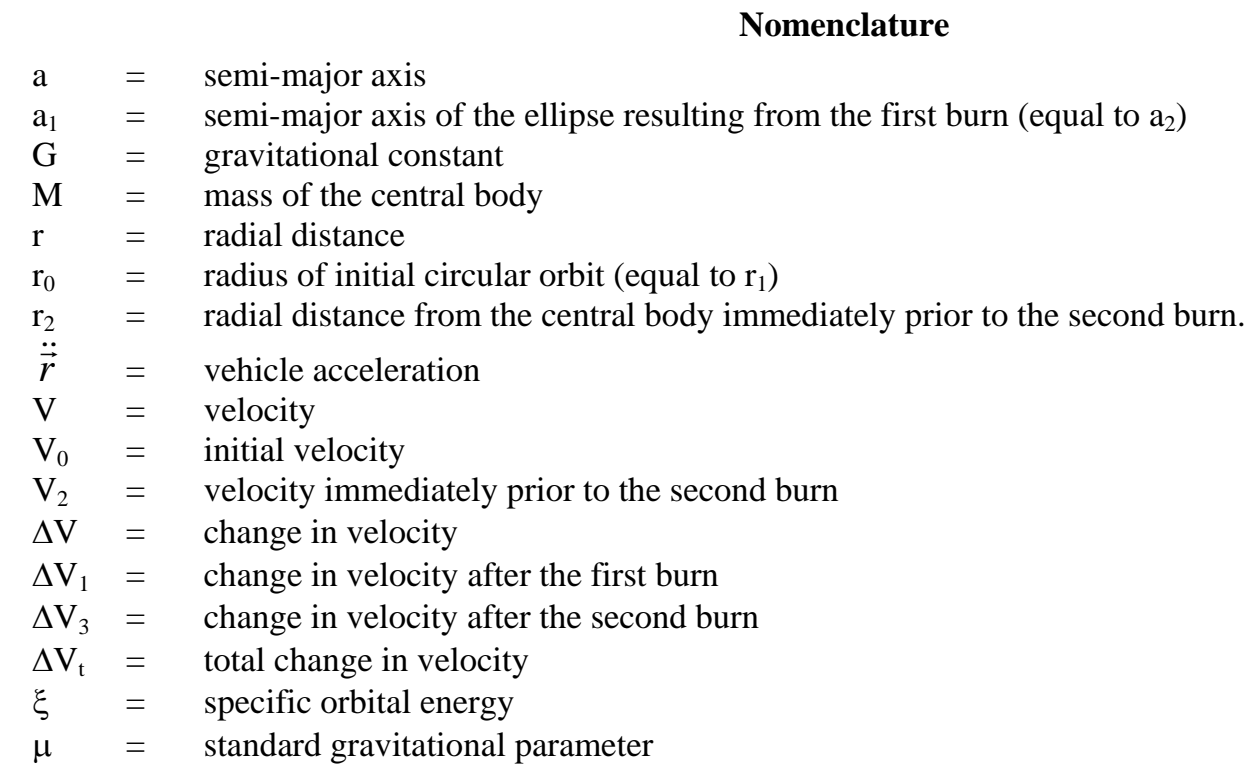

\footnotetext{
${ }^{1}$ Advanced Propulsion Technologist, National Aeronautics and Space Administration, George C. Marshall Space Flight Center, MSFC, AL 35812, AIAA Senior Member.

${ }^{2}$ Assistant Professor, University of Alabama in Huntsville, Mechanical and Aerospace Engineering Department, Huntsville, AL 35899.
} 


\section{Introduction}

In 1928 Oberth $^{1}$ suggested the option of a two-burn orbital maneuver that would, on the first burn, drop an orbiting spacecraft further down into the central body's gravity well, then a second burn would be performed to accelerate the spacecraft allowing it to escape the gravity well. This maneuver was not studied in detail by Oberth. Later, a brief analysis was done by Levin ${ }^{2}$. In this paper we present a detailed analysis of this two-burn maneuver including a comparison with a direct burn maneuver. Our analysis clearly shows that in some cases, the two-burn maneuver results in a higher specific mechanical energy as opposed to the direct burn. The analysis presented is a complete mathematical description without bias of the results to specific applications.

Two-body motion is of primary interest in most space trajectory applications as the vehicle is strongly affected by the planet (or star) it is orbiting compared with other distant astronomical bodies ${ }^{3}$. Similarly neglected are perturbations due to solar light pressure, atmospheric effects, spacecraft processes or gravitational harmonics due to the deviation from a uniform sphere of the central body. Neglecting the perturbing forces, the summation of forces on the vehicle is simply

$$
\ddot{\vec{r}}=\frac{\mu}{r^{2}}\left(\frac{\vec{r}}{r}\right) \text { with } \mu=G M \text {. }
$$

From the above equation the gravitational force is a function of the inverse square of this radius vector. In the Newtonian equation only the mass of the large body is considered since the mass of the vehicle is much smaller. Taking the dot product of equation (1) with respect to $\vec{r}$ yields

$$
\xi=\frac{V^{2}}{2}-\frac{\mu}{r}=-\frac{\mu}{2 a}
$$

where $\xi$ is referred to as the specific orbital energy of the vehicle. The orbital energy is the sum of the specific kinetic energy (the first term) and the specific potential energy (the second term). In deriving this equation it is convenient to assume that the reference line for potential energy is at infinity. The right hand side of the equation expresses specific orbital energy as a function of the orbits semi-major axis.

The equations above define the major parameters of a spacecraft in a coasting trajectory around a central body. In the operation of a spacecraft, changes in the orbit must be made to allow the spacecraft to travel to points of interest such as other planetary bodies. Turning on the spacecraft propulsion system, or performing a 'burn' in industry parlance, will place the vehicle in a new coasting trajectory once the burn is completed.

Conventional chemical propulsion systems are characterized as high thrust. The time to execute a burn is orders of magnitude lower than the overall mission time. High thrust burns are treated as impulsive, and so the equations above are applied to the new spacecraft velocity after the burn, which is sufficient to describe the new spacecraft orbit. The burn is quantified by another industry term, $\Delta V$, or "delta-vee", which is determined by the properties of the propulsion system, the types of propellants involved, and the amount of propellant used. Minimizing $\Delta V$ is the overriding performance objective in almost all spacecraft design endeavors. A smaller $\Delta V$ generally translates to a smaller spacecraft that is usually less expensive and more reliable to operate, simpler to design, and generally better able to meet mission objectives. It should be noted that the size of the spacecraft can be linked exponentially to the value of $\Delta V^{4}$ An example of high thrust propulsion systems are the conventional chemically powered liquid engines and solid motors seen on launch vehicles and spacecraft since the start of the space age.

Low thrust trajectories are characterized by propulsion systems that are on for large portions of the overall mission. These propulsion systems tend to be proportionally larger than those for high thrust systems, but also tend to require less propellant. Thus vehicles with low thrust propulsion systems are smaller and less massive than their high thrust counterparts in a wide variety of mission scenarios. Examples of low thrust propulsion systems are the nuclear and solar electric systems that have been strongly considered for a number of missions in recent years ${ }^{5}$. Additionally, solar electric propulsion can be found in previous NASA missions such as Deep Space 1 and are being used as station-keeping thrusters for a number of Earth orbiting satellites.

Because low thrust propulsion thrust duration is substantial compared to the mission time, the optimal direction of the propulsive thrust at each moment along the trajectory becomes important. Optimizing the "guidance 
schedule" of thrust direction and magnitude to limit the $\Delta V$ required and therefore the spacecraft size becomes an issue. Generally this is done by complex numerical integration of the equations of motion coupled with a variety of optimization routines. The optimization problem is difficult and there are a number of trajectory analysts that are dedicated on a daily basis to optimize these trajectories for missions of interest in space exploration.

Due to the computationally intensive nature of trajectory design, there has been strong interest since the 1950's to determine analytical approximations to low thrust trajectories that would give generally accurate results. A prevalent argument in the derivation of these approximations is that the thrust vector should always be aligned with the spacecraft's velocity vector. The reason can be seen by taking the time derivative of the kinetic energy equation, which is

$$
\frac{d}{d t} K E=\frac{d}{d t} \frac{V^{2}}{2}=\vec{V} \cdot \vec{a}
$$

Thus the instantaneous rate of change of kinetic energy is proportional to both acceleration and velocity. The local maximum is found when the spacecraft velocity and acceleration are parallel. However, as argued by Levin ${ }^{6}$, the spacecraft could accelerate in a different direction, forcing the spacecraft into a different orbit with a point of closest approach to the central body, the periapse, being closer to the central body than the original orbit. Examination of equation (2) shows that for a coasting orbit, the specific mechanical energy remains constant but the kinetic energy is traded for potential energy. At periapse kinetic energy is at a maximum and potential energy is at minimum, just like for any gravitational force dominated problem like a swinging pendulum or a ball in free flight. By equation (3), driving to a lower orbit and then accelerating at periapse would maximize the change in energy of the spacecraft for a given acceleration (and thrust).

This only describes the conversion of potential to kinetic energy. Understanding the infinite gain in specific mechanical energy requires discussion of another concept. One is tempted to expect only a finite amount of energy to be delivered to a spacecraft due to its latent chemical or nuclear energy. However the propellant has potential energy as well. Imagine that the spacecraft is propelled not by accelerating propellant, but by releasing a solid mass (of the same mass as the propellant it replaces) at high velocity, perhaps through use of a spring. The coiled spring in this case represents the latent energy of the propellant or propulsion system energy source. In the direct acceleration option both masses representing both burns would be released, propelling the vehicle into a higher orbit with periapse equal to the initial orbit. The spring loaded masses would be ejected into a lower orbit with apoapse equal to the initial orbit. The conservation of energy is not violated when one considers the change in kinetic energy of the spacecraft and the ejected masses. For the two-burn option, the first mass is ejected to slow the spacecraft and allow it to drop to a lower orbit. The ejected mass gains velocity and flies to a higher orbit about the central body. At periapse the spacecraft releases the second mass. This mass accelerates the vehicle and itself drops into a lower orbit. The derivations above show that the energy gained by the second mass ejection can greatly overcome the energy lost from the first mass ejection. The second mass can be left in a significantly lower orbit depending on the magnitude of the periapse radius. At the limit, the second mass is ejected from an infinitely small orbital radius, recovering an infinitely large change in kinetic energy for the spacecraft.

The discussed two-burn option could easily be confused with a gravity assist maneuver. However, the gravity assist maneuver is based on a massive body such as a planet dragging the spacecraft along for part of the spacecraft's trajectory. Momentum (and specific orbital energy) will be exchanged between the planet and spacecraft. The effect on the spacecraft is substantial, imparting in most cases a velocity change $(\Delta V)$ that could not be easily duplicated with current propulsion systems. The effect on the planet is minimal, due to its massive nature relative to the spacecraft. This momentum exchange is between an external body and the spacecraft and the exchange will occur even if no burn is made by the spacecraft. Conversely, a slingshot maneuver will not work unless there is a substantial burn at the periapse of the elliptical trajectory. The additional energy gained by the spacecraft is represented by the additional loss in specific energy by the propellant expended at the periapse burn. It does not represent a transfer of momentum from the central body to the spacecraft.

It should be noted that this principle is already in use in the context of a gravitational assist. It is well known that performing a burn maneuver at the periapse during a gravitational assist maneuver will enhance the overall $\Delta V$ gained from the maneuver. In fact the principle known as the Oberth Effect describes how a burn is most effective in accelerating a vehicle when it is conducted at the periapse of the spacecraft's orbit. ${ }^{7}$ 


\section{Theory for the two-burn maneuver}

As a thought experiment the authors considered a maneuver where the spacecraft would decelerate from an initial circular orbit with a single high thrust burn, dropping into an orbit with a lower specific mechanical energy. When the vehicle reaches the periapse of the new orbit it accelerates with another high thrust burn. The primary objective of this study is to determine if such a maneuver can produce a higher specific orbital energy than a direct burn from the initial circular orbit. A sketch of the maneuver is illustrated in Figure 1.

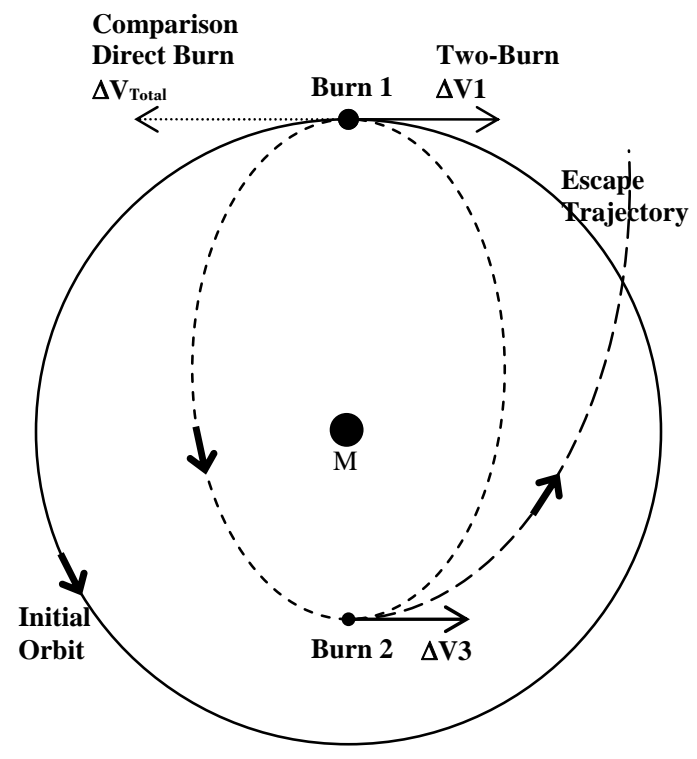

Figure 1. In the two-burn maneuver, the first burn decelerates the spacecraft resulting in a trajectory that takes it further into the gravity well of the central mass (this is opposed to a single direct burn which accelerates the spacecraft). The second burn is performed at the periapse of the new elliptical orbit and accelerates the spacecraft out of the gravity well of the central mass.

The primary purpose of this study is to derive an equation that compares the specific mechanical energy achieved after the second burn at periapse to that of a direct burn to escape. We begin by deriving an equation for the specific mechanical energy of the two-burn maneuver using the equations shown above. Starting with equation (2) and designating the second burn at periapse as $\Delta V_{3}$ the specific mechanical energy equation becomes

$$
\xi_{3}=\frac{\left(V_{2}+\Delta V_{3}\right)^{2}}{2}-\frac{\mu}{r_{3}}
$$

The second burn is made at periapse and is assumed to be an impulse burn. The periapse point is given by the definition of semi-major axis as $r_{2}=2 a_{1}-r_{1}$ where the semi-major axis can be calculated by the specific mechanical energy of the orbit before the $\Delta V_{3}$ burn to be $a_{1}=-\mu / 2 \xi_{1}$. With the substitution of the velocity immediately before the second burn, $\mathrm{V}_{2}$, using equation (2) the specific mechanical energy equation yields 


$$
\xi_{3}=\frac{1}{2}\left( \pm \sqrt{\frac{-2 \mu}{\frac{\mu}{\xi_{1}}+r_{1}}+2 \xi_{1}}+\Delta V_{3}\right)^{2}+\frac{\mu}{\frac{\mu}{\xi_{1}}+r_{1}}
$$

The specific energy of the orbit at position 1 can be defined from the conditions at position $1\left(\xi_{1}=V_{1}^{2} / 2-\mu / r_{1}\right)$. The specific mechanical energy equation then yields,

$$
\begin{gathered}
\xi_{3}=\frac{1}{2}\left( \pm \sqrt{\frac{4 \mu^{2}+r_{1}^{2} V_{1}^{4}-4 \mu r_{1} V_{1}^{2}}{r_{1}^{2} V_{1}^{2}}}+\Delta V_{3}\right)^{2}+ \\
\frac{\mu r_{1} V_{1}^{2}-2 \mu^{2}}{r_{1}^{2} V_{1}^{2}}
\end{gathered}
$$

For an initially circular orbit, the initial velocity can be expressed as $V_{0}=\sqrt{\mu / r_{0}}$. Again note that the burn the vehicle makes to depart the initial circular orbit $\left(\Delta V_{1}\right)$ is assumed to be instantaneous. Also note that the $\Delta V_{1}$ maneuver opposes the initial velocity of the spacecraft as shown in Figure 1. If we define the convenience variable $V_{1}=\sqrt{\mu / r_{0}}-\Delta V_{1}$, then with further algebra our final equation for the specific mechanical energy is,

$$
\xi_{3}=\left(V_{1}^{2}-2 \frac{\mu}{r_{0}}\right) \cdot\left(\frac{1}{2} \pm \frac{\Delta V_{3}}{V_{1}}\right)+\frac{\Delta V_{3}^{2}}{2}
$$

Here the final specific mechanical energy is a function of the radius of the initial orbit and the magnitude of the $\Delta V$ maneuvers only. Note that there is an asymptote when $V_{1}$ is zero, corresponding to $\Delta \mathrm{V}_{1}=\sqrt{\mu / \mathrm{r}_{0}}$.

We now revisit the direct burn option. The specific mechanical energy for the direct burn option is simpler to derive. Noting that the starting velocity has been defined previously and that the total change in velocity for the direct burn is $\Delta \mathrm{V}_{\text {Total }}=\Delta \mathrm{V}_{1}+\Delta \mathrm{V}_{3}$ the specific mechanical energy yields

$$
\xi_{\text {Direct }}=\frac{\left(\sqrt{\frac{\mu}{r_{0}}}+\Delta V_{1}+\Delta V_{3}\right)^{2}}{2}-\frac{\mu}{r_{0}}
$$

Setting the two energy equations equal to one another will yield the breakeven point between the direct option and the two-burn option 


$$
\begin{aligned}
& \frac{\left(\sqrt{\frac{\mu}{r_{0}}}+\Delta V_{1}+\Delta V_{3}\right)^{2}}{2}-\frac{\mu}{r_{0}}= \\
& \left(V_{1}^{2}-2 \frac{\mu}{r_{0}}\right) \cdot\left(\frac{1}{2} \pm \frac{\Delta V_{3}}{V_{1}}\right)+\frac{\Delta V_{3}^{2}}{2}
\end{aligned}
$$

Due to the plus/minus term which came from roots that developed during the derivation of the equations, there are two solutions. Taking the negative root results in,

$$
\Delta V_{1}+\Delta V_{3}=\sqrt{\frac{\mu}{r_{0}}} .
$$

This linear equation defines where the energy for the direct and two-burn maneuvers are equal. An additional line where this is true is when $\Delta \mathrm{V}_{1}=0$ as can be seen in the above energy equation. Taking the positive root results in the second equation,

$$
\begin{aligned}
& \left(\Delta V_{1}+\Delta V_{3}\right) \frac{\mu}{r_{0}}+ \\
& \Delta V_{1}\left(\Delta V_{3}-\Delta V_{1}\right) \sqrt{\frac{\mu}{r_{0}}}-\Delta V_{1}^{2} \Delta V_{3}=0
\end{aligned}
$$

To demonstrate when the two-burn option will produce a greater specific mechanical energy over that of the direct burn option, we present Figures 2 - 5. Figures 2 and 4 show the negative root from the above equations and Figures 3 and 5 show the positive root. Figures 2 and 3 are for an initial Low Earth Orbit (LEO) for a trajectory around the Earth and Figures 4 and 5 are for an initial orbit of one astronomical unit (AU) for a trajectory around the Sun. In each of these plots, the top figure gives $\Delta \mathrm{V}_{1}$ versus specific mechanical energy for a constant $\Delta \mathrm{V}_{3}$. The three vertical lines indicate the two cross over points and the vertical asymptote mentioned above. The lower figure plots the above two equations for $\Delta \mathrm{V}_{1}$ versus $\Delta \mathrm{V}_{3}$. This plot shows where the direct burn and two-burn options will have the same specific mechanical energy. The two vertical lines show the two cross over points.

For the two-burn maneuver to have a higher specific mechanical energy, $\Delta \mathrm{V}_{1}$ must fall in specific regions. If one considers the three vertical lines in the top plots to divide the plot into four regions, and we number those regions 1-4 from left to right, the 2-burn option will have a higher specific mechanical energy in regions 1 and 3 . In the other regions, the direct burn maneuver will have a higher specific mechanical energy. Looking at the Earth orbital figures and the negative root option, this means the 2-burn $\Delta \mathrm{V}_{1}$ must meet the following criteria; $\Delta \mathrm{V}_{1}<-0.618 \mathrm{~V}_{0}$ or $0<\Delta \mathrm{V}_{1}<1.618 \mathrm{~V}_{0}$. For the positive root, the $\Delta \mathrm{V}_{1}$ criteria is $\Delta \mathrm{V}_{1}<\mathrm{x}_{1}$ where $\mathrm{x}_{1}$ is the negative root of $\left(-\mathrm{V}_{0}-\Delta \mathrm{V}_{3}\right) \Delta \mathrm{V}_{1}^{2}+\left(\mathrm{V}_{0}{ }^{2}+\mathrm{V}_{0} \Delta \mathrm{V}_{3}\right) \Delta \mathrm{V}_{1}+\mathrm{V}_{0}{ }^{2} \Delta \mathrm{V}_{3}=0$ or $\mathrm{V}_{0}<\Delta \mathrm{V}_{1}<\mathrm{x}_{2}$ where $\mathrm{x}_{2}$ is the positive root of the above equation. Note that the $\mathrm{x}_{2}$ point may appear either before or after the vertical asymptote depending on the value of $\Delta \mathrm{V}_{3}$.

With respect to the total $\Delta V$ budget, Figure 6 shows the specific mechanical energy related to $\Delta V_{1}$. This figure was calculated for the same $1 \mathrm{AU}$ orbit as Figures 4 and used the negative root solution. The figure re- 
emphasizes the effect when $\Delta \mathrm{V}_{1}=\mathrm{V}_{0}$. The asymptote at this point puts the specific mechanical energy at positive or negative infinity depending on the value of $\Delta \mathrm{V}_{1}$. This is true for any total $\Delta V$ budget.

Figure 7 emphasizes the amount by which the two-burn maneuver can exceed the direct maneuver in producing escape velocity. In Figure 7 it is clear that the two-burn maneuver does not gain relative to a direct burn unless the total $\Delta V$ exceeds that of the initial velocity. The figure also shows that the maneuver can gain substantially relative to the direct burn under the right circumstances. The plot also shows the first $\Delta V$ required to drop perihelion to that of Mercury and Venus' average orbital distance and that to drop perihelion to within 3 solar radii. It is evident that the spacecraft must come very close to Sol in this scenario to realize significant gains in $V_{\text {inf. }}$ Additionally the larger the entire $\Delta V$ budget is, the further perihelion can be to realize the same gain in $V_{\text {inf }}$.
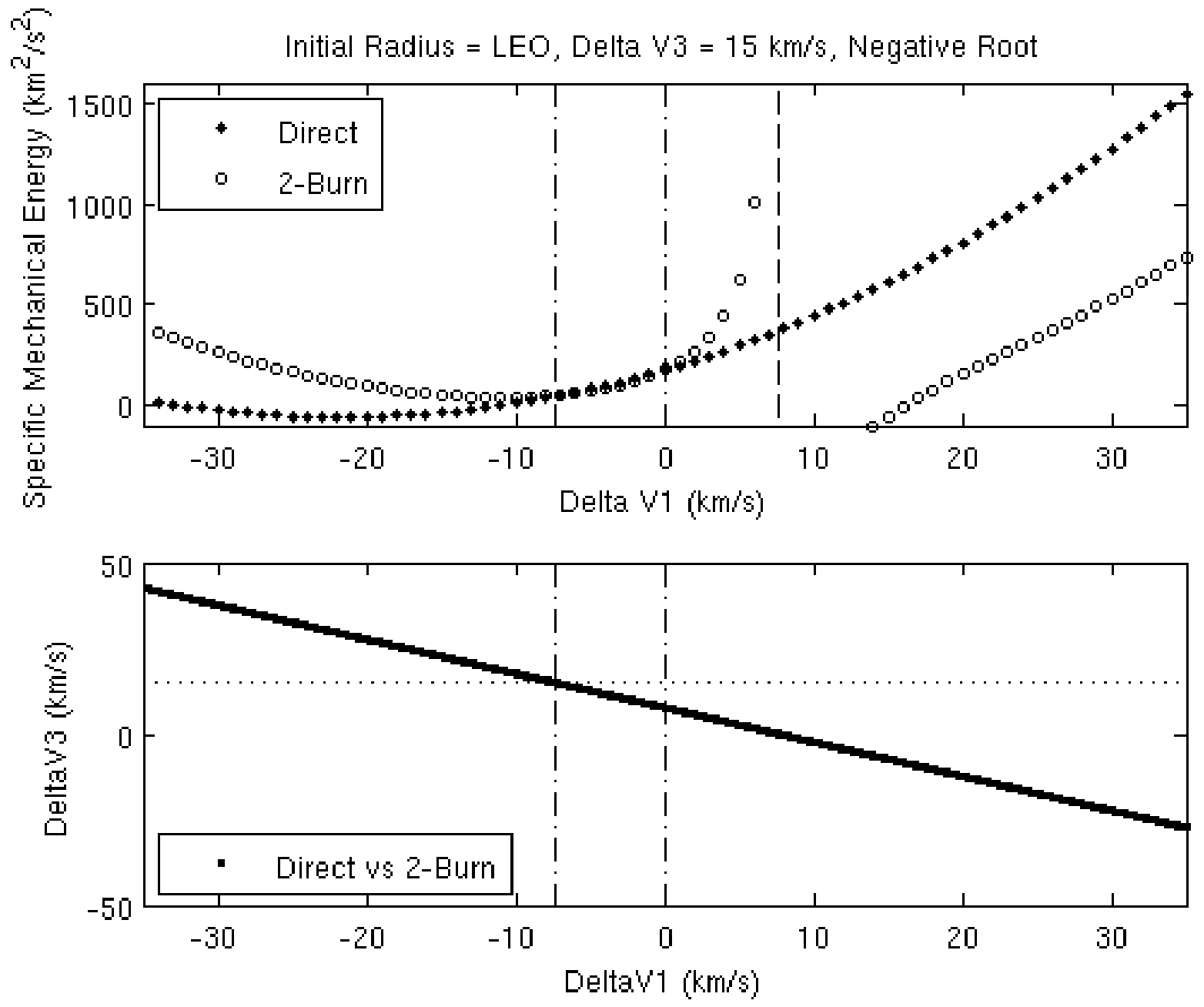

Figure 2. Comparison between a direct burn and the negative root of the two-burn maneuvers around the Earth from an initial orbit of $\mathbf{4 0 0} \mathbf{~ k m}$ above the Earth (LEO). In the top figure $\Delta \mathrm{V}_{3}=15 \mathrm{~km} / \mathrm{s}$ and the two-burn maneuver shows a higher specific mechanical energy in two of the four regions. The lower figure shows two points where the specific mechanical energy for the two maneuvers will be equal, the crossing of the horizontal and sloped lines and at the vertical line, $\Delta \mathrm{V}_{1}=0 \mathrm{~km} / \mathrm{s}$. 

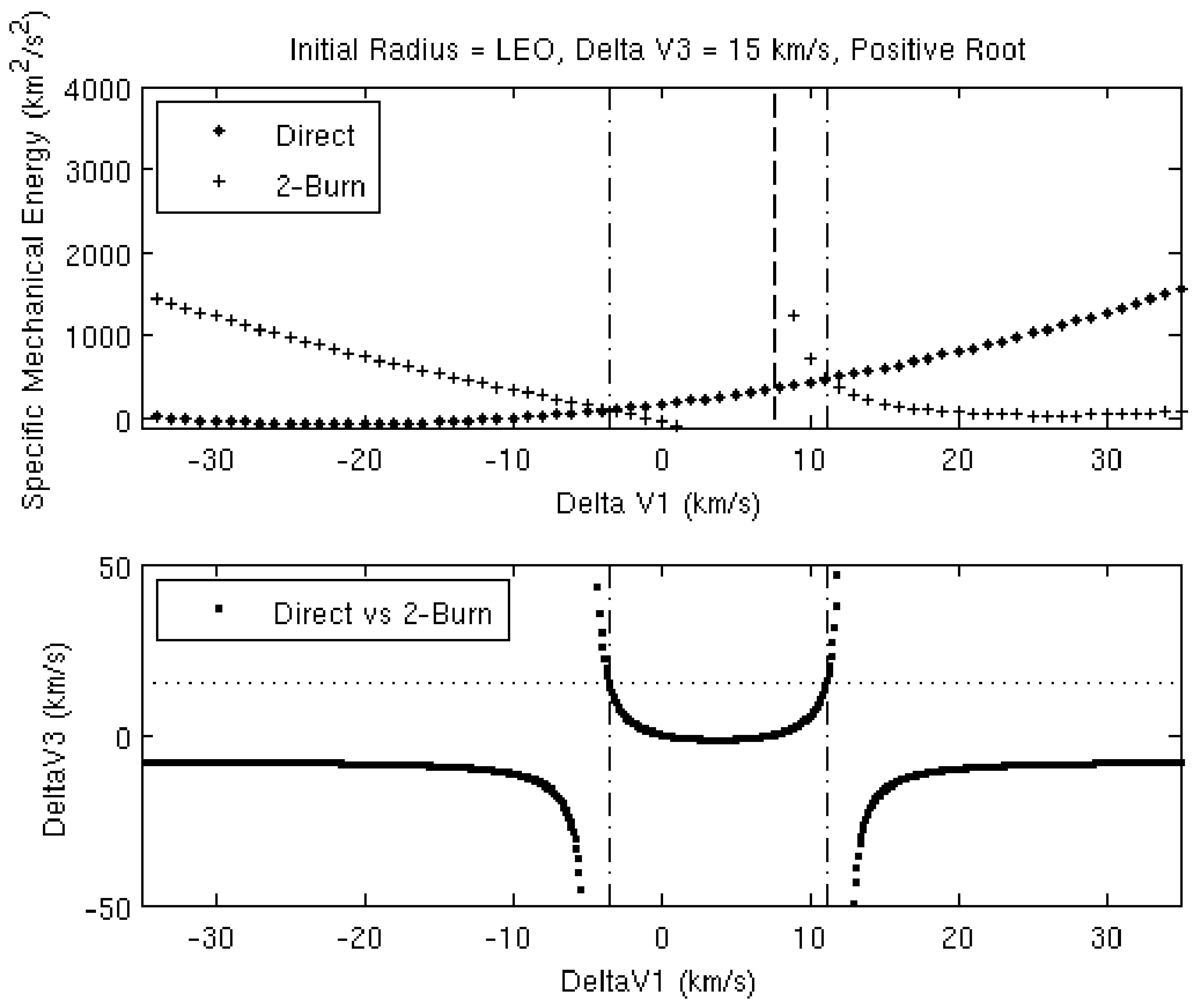

Figure 3. Comparison between a direct burn and the positive root of the two-burn maneuvers around the Earth from an initial orbit of $400 \mathrm{~km}$ above the Earth (LEO). In the top figure $\Delta \mathrm{V}_{3}=15 \mathrm{~km} / \mathrm{s}$ and the two-burn maneuver shows a higher specific mechanical energy in two of the four regions. The lower figure shows two points where the specific mechanical energy for the two maneuvers will be equal, at the crossing of the horizontal line and the non-linear function.

For instance, assuming that the spacecraft can survive a close approach to the sun of 3 solar radii and a total $\Delta V$ budget of $40 \mathrm{~km} / \mathrm{sec}$ the slingshot maneuver can produce a change in specific orbital energy approximately three times that of the direct maneuver. Survival of a three solar radii approach is the goal of Solar Probe+ mission ${ }^{8}$, so there is good expectation that this goal is within current technological limits. There is also the possibility of using the two-burn maneuver not only for escape but also for orbit raising missions. However the maneuver as shown is not more efficient than a Hohmann maneuver. This is evident by considering the concept of escape velocity. Given a spacecraft at a certain orbital radius the minimum velocity that will attain escape from the central body is

$$
V_{\text {esc }}=\sqrt{\frac{2 \mu}{r_{0}}}
$$

Comparing the escape velocity to initial circular velocity plus the budget allowed for a maneuver gives,

$$
\sqrt{\frac{\mu}{r_{0}}}+\Delta V_{\text {tot }}=\sqrt{\frac{2 \mu}{r_{0}}}
$$



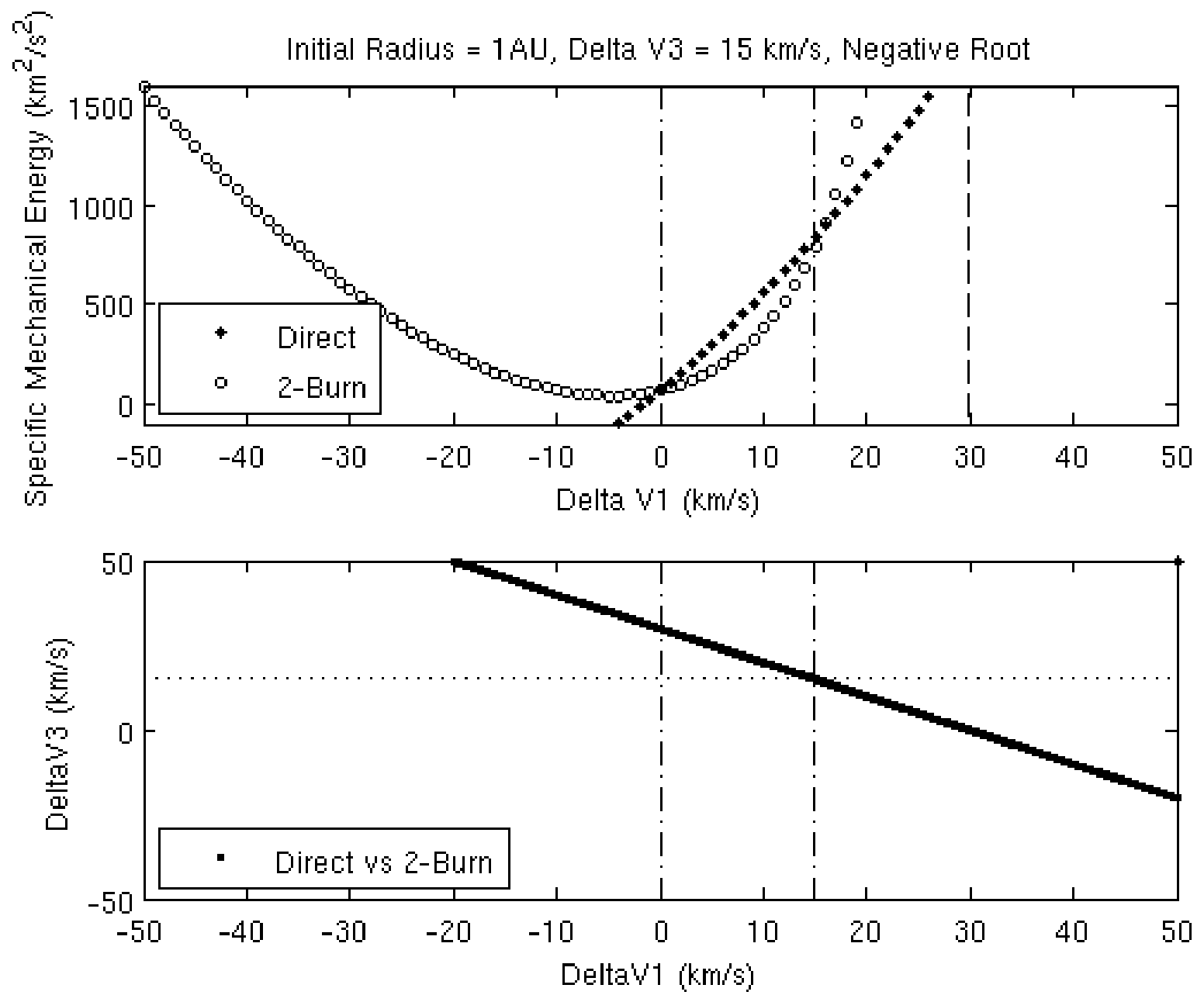

Figure 4. Comparison between a direct burn and the negative root of the two-burn maneuvers around the Sun from an initial orbit of $1 \mathrm{AU}$. In the top figure $\Delta \mathrm{V}_{3}=15 \mathrm{~km} / \mathrm{s}$ and the two-burn maneuver shows a higher specific mechanical energy in two of the four regions. The lower figure shows two points where the specific mechanical energy for the two maneuvers will be equal, the crossing of the horizontal and sloped lines and at the vertical line, $\Delta \mathrm{V}_{1}=0 \mathrm{~km} / \mathrm{s}$.

Since the two-burn maneuver requires that the $\Delta V$ budget exceeds the circular velocity the left hand side of the equation will always exceed escape velocity. Thus the two-burn maneuver is not as efficient as that for a Hohmann transfer. However, frequently a mission requires a fast transfer from one orbit to another, faster than the Hohmann transfer produces. Crewed missions and robotic probes to deep space make waiting for Hohmann transfers prohibitive. Thus the two-burn maneuver could be compared to a semi-tangential trajectory. In this scenario there are some instances where the two-burn maneuver proves more effective. More analysis is indicated in this area.

It should be noted that the analysis in figures 6 and 7 assume a starting position of an orbit around the sun at earth's distance (1 AU). The premise of this two-burn maneuver is that burns should be done as low in the gravity well as possible. In a realistic situation the first burn, intending to slow the spacecraft so it will drop towards the sun, would be done in Low Earth Orbit. The benefit is that the first burn is done deep in the gravity well of Earth making it less expensive to drop towards the sun. The difference can be significant, and the results above for starting points in orbit around the sun are therefore conservative. 

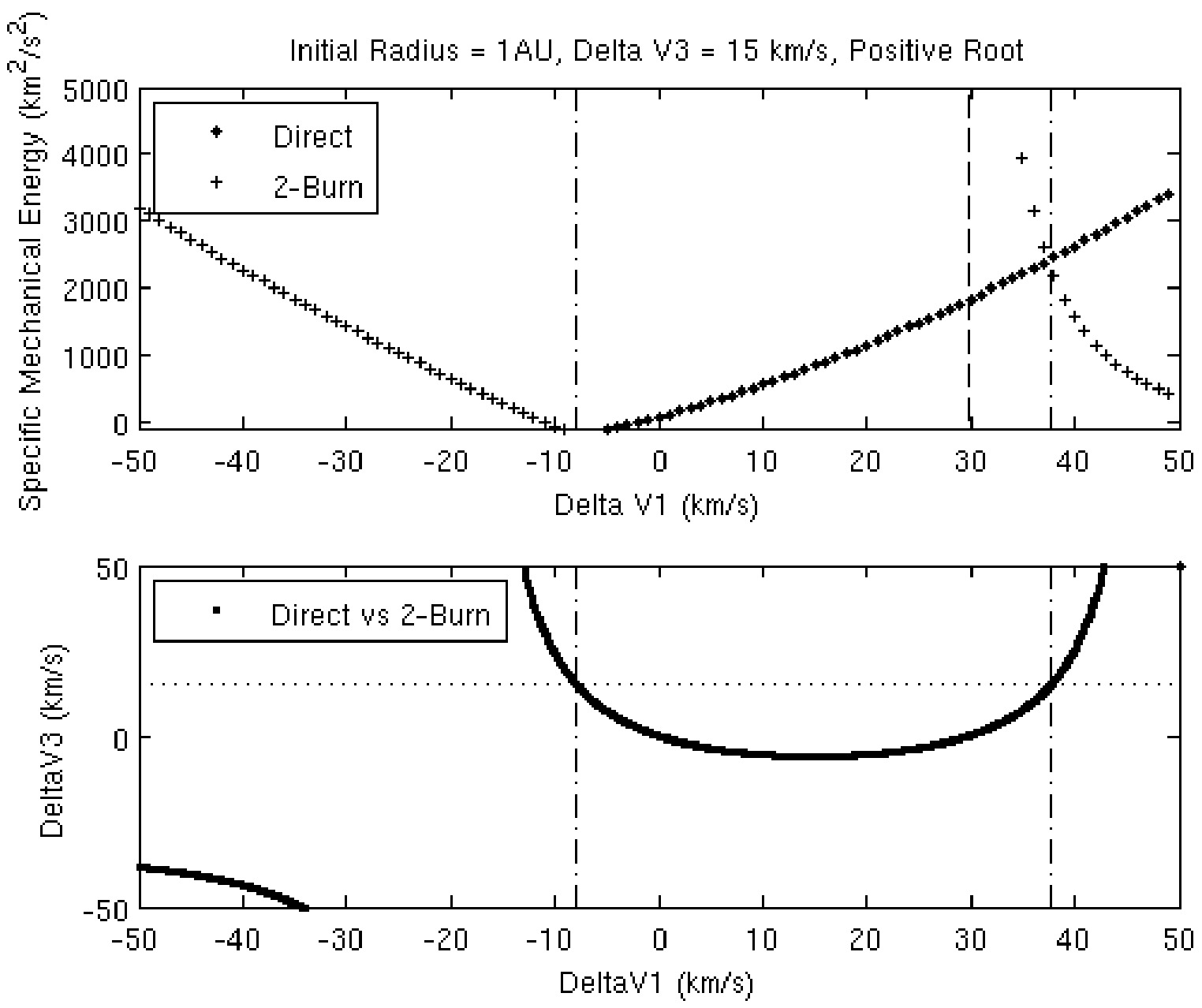

Figure 5. Comparison between a direct burn and the positive root of the two-burn maneuvers around the Sun from an initial orbit of $1 \mathrm{AU}$. In the top figure $\Delta \mathrm{V}_{3}=15 \mathrm{~km} / \mathrm{s}$ and the two-burn maneuver shows a higher specific mechanical energy in two of the four regions. The lower figure shows two points where the specific mechanical energy for the two maneuvers will be equal, at the crossing of the horizontal line and the nonlinear function.

\section{Conclusions}

The two-burn option can produce a greater specific mechanical energy for a given $\Delta V$ budget than a direct burn but only when the total budget exceeds the initial velocity in the initial orbit. So the $\Delta V$ budget must be considerable before the slingshot maneuver is worthwhile. For instance starting from a circular orbit around the sun at a distance equal to earth's orbit, the $\Delta V$ budget equal to the initial circular velocity is sufficient to completely escape the solar system with a $V_{\infty}$ of about $17.5 \mathrm{~km} / \mathrm{sec}$. However the $\Delta V$ budget is well within the range of many missions of interest to NASA. For instance the interstellar precursor mission presents the challenge of traveling 1000 astronomical units (AU) within 50 years, the career lifetime of the average engineer or scientist. The escape velocity above will deliver a spacecraft to the required distance in over 110 years so clearly a slingshot maneuver would be useful for this mission. Other deep space missions to the outer planets, Kuiper Belt, Oort Cloud, and heliopause would similarly be enhanced by use of this maneuver.

Finally a class of mission that has received attention by NASA in recent years is the deflection or fragmentation of asteroids and comets that are on a collision course with Earth. The $\Delta V$ imparted to an oncoming asteroid is very low, on the order of $1-100 \mathrm{~cm} / \mathrm{sec}^{9}$. This $\Delta V$ is sufficient to deflect most asteroids provided that 
the impulse is applied to the asteroid early enough. Current deflection methods require 2-50 years between application of the impulse and the projected collision date. Therefore the device that will impart the impulse to the asteroid must intercept or rendezvous with the asteroid with all haste. Given the above the $\Delta V$ requirement to intercept an incoming asteroid is generally on the order of $10-30 \mathrm{~km} / \mathrm{s}^{10}$. The $\Delta V$ requirement to rendezvous can be as high as $70 \mathrm{~km} / \mathrm{sec}$. Both values are well within the range necessary to make the two-burn maneuver economical.

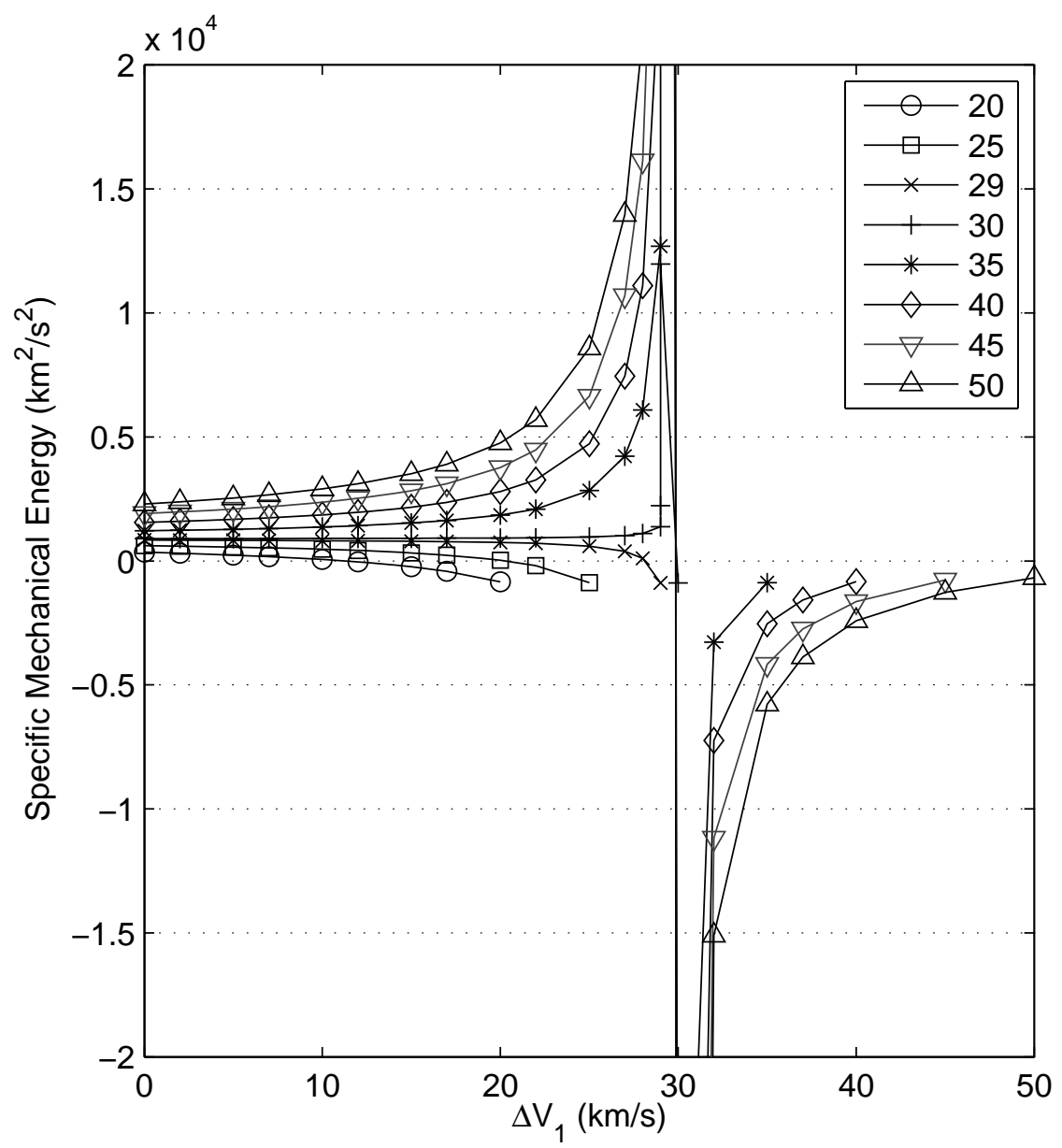

Figure 6. The specific mechanical energy of the negative root two-burn maneuver, versus $\Delta \mathrm{V}_{1}$ for varying total $\Delta \mathrm{V}$ budgets. The vertical asymptote is at the initial orbital velocity $(29.78 \mathrm{~km} / \mathrm{s})$. Values represent a solar orbit starting at $1 \mathrm{AU}$ (Earth-like) orbit.

This paper started by examining the concept that acceleration along the velocity vector would result in an optimal acceleration of the spacecraft. While acceleration along the velocity vector is locally optimal it turns out that there is a special maneuver that in certain cases will outperform the "optimal" cross product acceleration by actually decelerating the vehicle and accelerating it when it reaches periapse. It is presented here as an important maneuver to be considered for high $\Delta V$ missions such as interstellar precursor or similar deep space missions and potentially crewed round trip missions to Mars and beyond. This has profound implications for future space exploration. Being able to use in-situ resources to create propellants or even construct vehicles on the moon has even greater importance now that the two-burn maneuver can be used to substantially reduce the propulsive requirements for deep space missions. 
The $\Delta V$ required to complete a mission to Mars and return is $12-13 \mathrm{~km} / \mathrm{sec}$ for Hohmann transfers. However, radiation exposure, crew supplies and crew mental health issues have forced vehicle designers to look at much higher $\Delta V$ missions that can reduce trip times and mission risk. Mission studies for crewed missions to Mars with a limiting total trip time of 2 years of less have $\Delta V$ requirements above $20 \mathrm{~km} / \mathrm{sec}$. Therefore it is possible that the new option could have application in orbit raising maneuvers where limiting mission time is critical. A proof of principle calculation shows significant gains in performance for crewed missions to Mars using this maneuver. This gain is predicated on the hope that water ice will be found on the moon and can successfully be turned into useable propellant. Given this assumption the two-burn maneuver can reduce vehicle size by up to half, or decrease mission time by half. The former dramatically reduces the cost of a Mars trip, while the latter reduces the risk to crew.

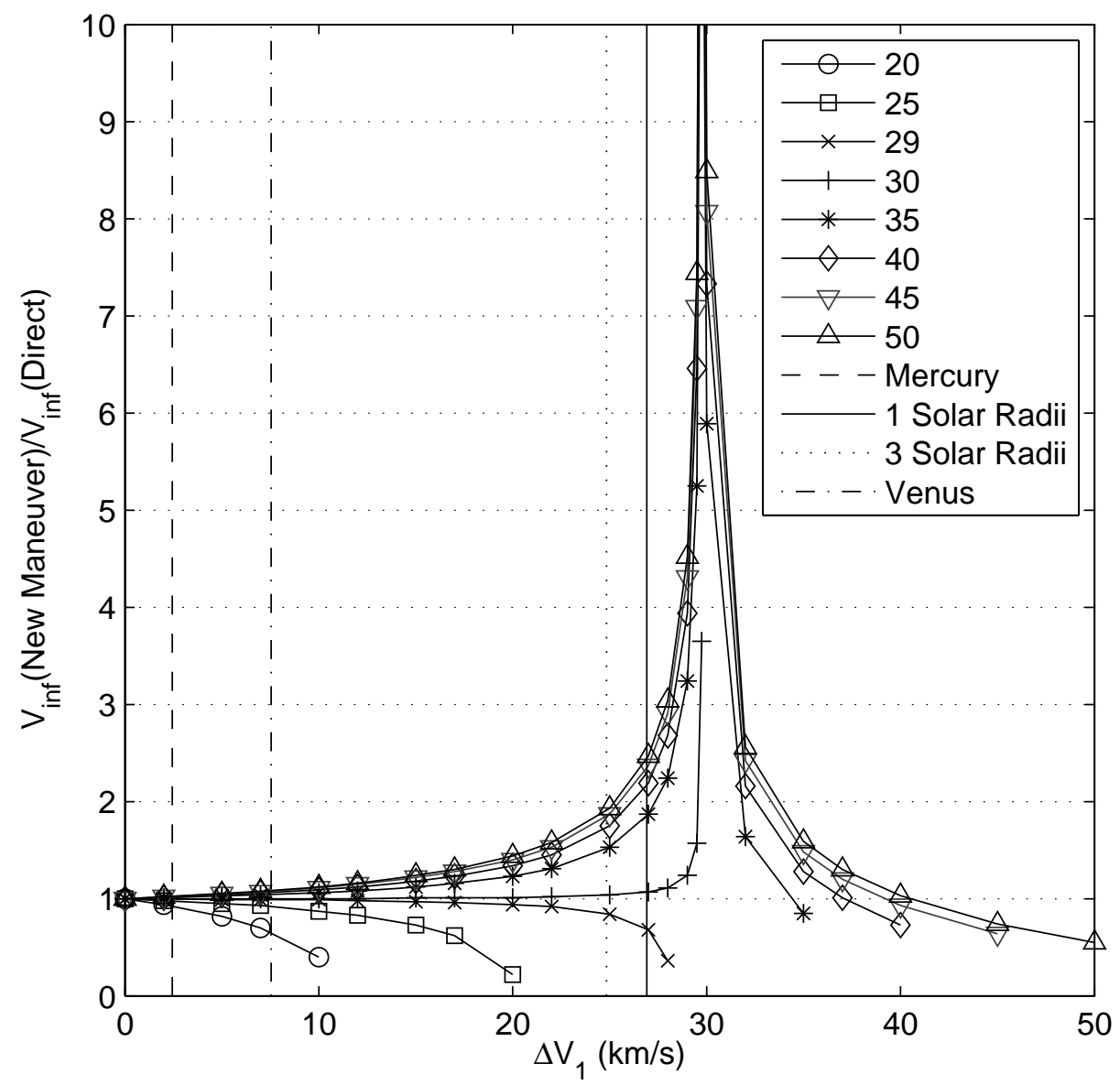

Figure 7. Ratio of $V_{\text {inf }}$ for the two-burn maneuver to the $V_{\text {inf }}$ attained through a direct burn over a range of total $\Delta \mathrm{V}$ budgets. The negative root solution was used for this figure. The vertical asymptote is at the initial orbital velocity $(29.78 \mathrm{~km} / \mathrm{s})$. The values represent a solar orbit starting at $1 \mathrm{AU}$ (Earth-like orbit).

The two-burn maneuver shows considerable promise to enable a variety of scientific and exploration missions in deep space. The authors believe that this two-burn maneuver could have as large of an impact on space exploration as the gravity assist. Developed at the very beginning of the space program, the gravity assist enabled missions from Voyager to Cassini to visit the planets of the solar system using technologies that were then available. 
Clearly without the gravity assist those technologies would have been inadequate to explore much of the solar system outside of the moon and Mars. Similarly the two-burn maneuver provides a method for exploration of the boundary of the solar system and interstellar space using today's technologies and technologies of the near future. Such missions are difficult to conceive without considering the advantages of alternative maneuvers.

\section{Acknowledgements}

The authors would like to acknowledge Dr. Clark Hawk as a mentor, colleague and friend. Without his support and guidance, this work would not have been possible.

\section{References}

\footnotetext{
${ }^{1}$ Oberth, Hermann, Ways to Spaceflight, translated from German in NASA TT F-622.

${ }^{2}$ Levin, D. F.., “Escape to Infinity from Circular Orbits”,Journal of the British Interplanetary Society, Vol. 12, No. 2, March 1953, pp.68-71.

${ }^{3}$ Vallado, D. A., Fundamentals of Astrodynamics and Applications, Microcosm Press, El Segundo, CA, 2001

${ }^{4}$ Sutton, G. P., Bilbarz, O., Rocket Propulsion Elements, John Wiley and Sons, Inc., 2001.

${ }^{5}$ Lawrence, T. J., Witter, Jonathan K., Humble, Ronald W., “Nuclear Rocket Propulsion Systems”, Chapter 8 in Space Propulsion Analysis and Design, The McGraw-Hill Companies, New York, 1995.

${ }^{6}$ Levin, E., "Low Acceleration Transfer Orbits", Section 9.1 in Handbook of Astronautical Engineering, edited by Heinz Hermann Koelle, McGraw-Hill book Company, New York, 1961.

${ }^{7}$ Oberth Effect, http://en.wikipedia.org/wiki/Oberth_effect, accessed February 6, 2009.

${ }^{8}$ Danzler, A. A., “Solar Probe+, “Mission Engineering Study Report”, NASA’s Heliophysics Division, March 10, 2008.

${ }^{9}$ Near-Earth Object Survey and Deflection Analysis of Alternatives, Report to Congress, NASA, March, 2007.

${ }^{10}$ Adams R. B., Alexander, R., Bonometti, J., Chapman, j., Fincher, S., Hopkins, R., Kalkstein, M., Polsgrove, T., Statham, G., White, S., Survey of Technologies Relevant to Defense from Near-Earth Objects, NASA, TP-2004-213089.
} 